\title{
Rudolf Behrens, Jörn Steigerwald (dir.), Aufklärung \\ und Imagination in Frankreich (1675-1810)
}

\section{Claire Gantet}

\section{OpenEdition}

\section{Journals}

Édition électronique

URL : http://journals.openedition.org/ifha/10221

ISSN : 2198-8943

\section{Éditeur}

IFRA - Institut franco-allemand (sciences historiques et sociales)

\section{Référence électronique}

Claire Gantet, « Rudolf Behrens, Jörn Steigerwald (dir.), Aufklärung und Imagination in Frankreich (16751810) », Revue de l'IFHA [En ligne], Date de recension, mis en ligne le 26 juillet 2019, consulté le 23 septembre 2020. URL : http://journals.openedition.org/ifha/10221

Ce document a été généré automatiquement le 23 septembre 2020

(CIFHA 


\title{
Rudolf Behrens, Jörn Steigerwald (dir.), Aufklärung und Imagination in
} Frankreich (1675-1810)

\author{
Claire Gantet
}

\section{RÉFÉRENCE}

Rudolf Behrens, Jörn Steigerwald (dir.), Aufklärung und Imagination in Frankreich (16751810). Anthologie und Analyse, Berlin/Boston : De Gruyter (Hallesche Beiträge zur Europäischen Aufklärung, 54), 2016, X-660 p., 139,95€ 
Si l'imagination est depuis toujours une faculté innée de l'être humain, elle ne relève pas des invariants anthropologiques. L'interprétation de sa nature - cognitive ou déviante -, de ses relations avec les autres sens internesentendement, et plus encore mémoire -, de ses liens avec le monde extérieur général et le corps individuel particulier, de ses effets enfin-aberrants ou esthétiques - sont l'objet d'une histoire aussi essentielle que complexe. C'est à retracer des étapes de cette histoire mal connue au XVIII ${ }^{e}$ siècle, dans des sources rédigées en français, que s'attellent le romaniste Rudolf Behrens et Jörn Steigerwald, professeur de littérature comparée à l'université de Paderborn. Le projet a été longuement mûri et est livré

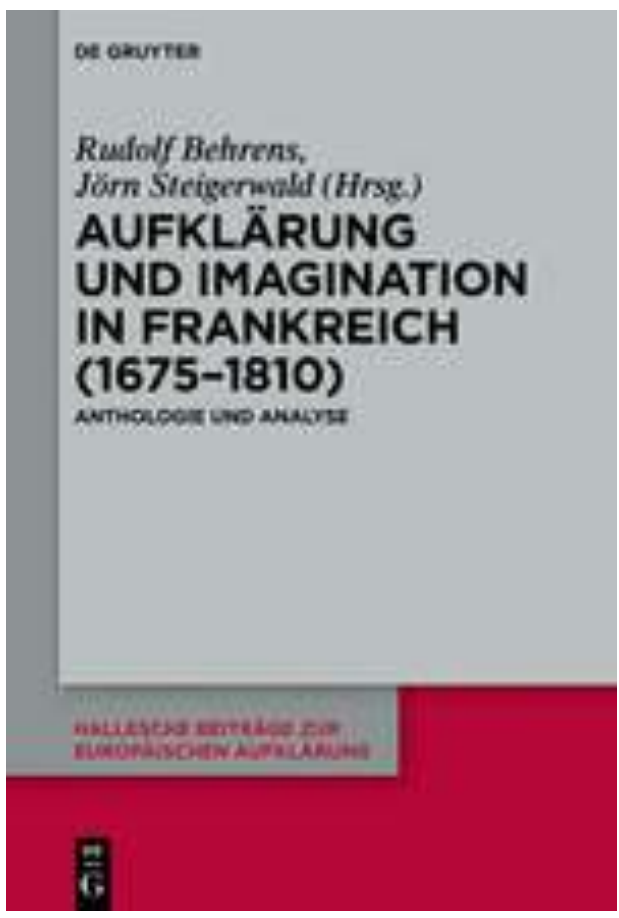
au public de façon souveraine. Après une présentation du programme de travail en introduction, il consiste en 26 chapitres ordonnés par auteurs brièvement caractérisés et illustrés d'extraits originaux, de façon chronologique. Le XVIII siècle francophone est compris au sens large chronologiquement et spatialement, puisque le volume s'ouvre sur Malebranche (1674/1675) et se clôt sur le Suisse Charles-Victor de Bonstetten (1807). Les contributions sont rédigées par des membres de l'équipe des directeurs, Jörn Steigerwald ayant lui-même rédigé pas moins de dix chapitres.

Dès l'Antiquité, les relations de l'imagination avec la mémoire furent débattues. Selon Aristote en effet, la phantasia représentait de façon visuelle le contenu des pensées (les phantasmata). La force d'illusion potentiellement contenue par l'action de l'imagination suscita de nombreuses réflexions tout au long du Moyen Âge, où l'on distingua une imagination passive ou reproductrice (des impressions vécues), d'une imagination active ou productrice (combinant les impressions passées). En réponse à l'héritage intellectuel arabe et à la pensée de Galien, on forma aussi un corps d'idées sur le substrat corporel de l'action de l'imagination. Peu à peu s'esquissa ainsi une aspiration majeure au XVIII e siècle : cerner les pouvoirs de l'imagination pour réguler son action et lutter contre l'«enthousiame débridé», la Schwärmerei, et contre la superstition. Tandis que certains lettrés de la Renaissance, de Marsile Ficin à Montaigne en passant par Giordano Bruno, sondaient son action en termes de sémiotique, Gianfrancesco Pico della Mirandola suggéra dès 1600 la difficulté à discipliner le travail induit par l'imagination, issu de circonstances extérieures et de dispositions corporelles.

Vers 1700, divers courants se dessinent. Le premier, marqué par Malebranche (mais influençant encore Jean-Jacques Rousseau et des auteurs du début du XIX ${ }^{\mathrm{e}}$ siècle), campe sur une acception rationaliste des facultés de l'âme: l'imagination est vue comme une source d'errements qu'il convient de chercher à endiguer. Les images intérieures sont considérées comme portées par des esprits animaux parcourant les nerfs reliant les sens extérieurs à l'âme; elles sont sémantiquement floues, peu 
distinctes, chargées d'affects (ou passions de l'âme) et confuses, dépendant de dispositions corporelles telles que le sexe, l'âge, etc. Dans la Recherche de la vérité, Malebranche suggère surtout le caractère contagieux de l'imagination lorsque ces images imprécises, arbitraires et non réelles sont mises en discours et en images, entrant ainsi dans la communication interpersonnelle. Une telle disposition à la contagion hante le discours médical sur les pathologies que l'on y rattache (mélancolie, hystérie, délires, onanisme, etc.) jusqu'à la fin du XVIII ${ }^{\mathrm{e}}$ siècle.

Face à cette défiance pérenne de l'imagination s'affirment deux tendances nettement plus positives. La première adopte une perspective descriptive (" phénoménologique »), caractérisant Fénelon, Rousseau (là aussi), l'abbé Pluche et Charles-Victor de Bonstetten, un proche de Madame de Staël. La seconde, partant d'un point de vue physiologique et matérialiste, singularise les œuvres de La Mettrie, de Diderot et du marquis de Sade. Différentes anthropologies et sensibilités scientifiques coexistent et s'affrontent donc au XVIII ${ }^{\mathrm{e}}$ siècle, inspirées par le cartésianisme, le sensualisme, le déisme et le matérialisme notamment, et alternant les présentations scientifiques, de vulgarisation, et littéraires. Un mouvement de fond : la substitution d'une interrogation « horizontale », physiologique et épistémique, à une approche verticale, théologique et ontologique, prélude à une forte différenciation de la notion d'imagination.

On connait l'influence de la psychologie anglaise, en particulier des textes de Locke; l'ouvrage met en lumière, à côté de lui, l'ascendant de Shaftesbury et de Joseph Addison sur le sensualisme et notamment Condillac. En l'absence d'une réelle définition de la matière sensible, l'imagination sert chez La Mettrie de commun dénominateur aux processus de perception, de connaissance et de combinaison d'idées. Si La Mettrie a été peu cité par ses contemporains, ses réflexions provocantes ont néanmoins porté Diderot à tenter de mieux caractériser l'imagination, en s'inspirant des découvertes médicales récentes et en prolongeant la réflexion sur le plan esthétique. Tous les hommes de science se penchent sur l'imagination au milieu du XVIII siècle. Tandis que Buffon, dans son Histoire naturelle, fait de l'imagination le signe de la supériorité de l'être humain sur tous les autres êtres vivants, l'abbé Pluche mise sur la connaissance sensorielle et l'imagination dans son Spectacle de la nature (9 vol., 1732-1750): l'imagination y est la marque de la nature créatrice de l'être humain dont dépend toute science et présentation de la science. Dès le milieu du XVIII ${ }^{e}$ siècle, on la valorise comme puissance de création esthétique : les romantiques, ici, ne sont que des héritiers des Lumières. Le discours médical s'avère là aussi déterminant. La "découverte » de l'hygiène individuelle et sociale induit un nouveau débat sur la sexualité, sur la procréation et sur la nature de la femme et des maladies pensées comme spécifiques, et une discussion publique sur le mode de vie des savants.

L'ouvrage met en lumière à la fois la multiplicité des points de vue sur l'imagination et des tendances de fond, telles que l'importance accrue de la physiologie et de l'esthétique dans la nouvelle anthropologie du milieu du XVIII ${ }^{\text {e }}$ siècle - on comprendra alors que de nombreux écrivains étaient médecins. Il rappelle des textes connus mais exhume surtout des auteurs largement oubliés et pourtant essentiels, tels Antoine Le Camus, Guillaume-Lambert Godart ou Antoine-Joseph Pernety. Il manifeste aussi à quel point le débat francophone ne saurait être enserré dans les frontières de l'« hexagone ». On ne regrette que l'absence d'index qui dépare l'ouvrage, et l'absence complète d'une discussion de la "psychologisation ». À l'issue de la lecture de ce livre, qui est une somme et un ouvrage de référence indispensable, on souhaite que le projet 
soit poursuivi et amplifié en une coopération franco-allemande, voire franco-germanoanglaise.

INDEX

Thèmes : Histoire de la culture, Histoire des idées, Manuels/Ouvrages généraux/Outils de travail Index chronologique : Période moderne

\section{AUTEURS}

\section{CLAIRE GANTET}

Université de Fribourg (Suisse)/Universität Freiburg (Schweiz) 